\title{
OLED Fabrication by Using a Novel Planar Evaporation Technique
}

\author{
Fu-Ching Tung, ${ }^{1,2}$ Yi-Shan Wang, ${ }^{2}$ Shih-Hsiang Lai, ${ }^{2}$ Chien-Chih $\mathrm{Chen},{ }^{2}$ Szu-Hao Chen, \\ Ching-Chiun Wang, ${ }^{2}$ Jwo-Huei Jou, ${ }^{1}$ and Sun-Zen Chen $^{3}$ \\ ${ }^{1}$ Department of Materials Science and Engineering, National Tsing Hua University, Hsinchu 30013, Taiwan \\ ${ }^{2}$ Mechanical and Systems Research Laboratories, Industrial Technology Research Institute, Hsinchu 31040, Taiwan \\ ${ }^{3}$ Center for Nanotechnology, Materials Science, and Microsystems, National Tsing Hua University, Hsinchu 30013, Taiwan
}

Correspondence should be addressed to Fu-Ching Tung; fctung@itri.org.tw and Jwo-Huei Jou; jjou@mx.nthu.edu.tw

Received 13 March 2014; Revised 15 May 2014; Accepted 23 May 2014; Published 22 June 2014

Academic Editor: Liang-Sheng Liao

Copyright (C) 2014 Fu-Ching Tung et al. This is an open access article distributed under the Creative Commons Attribution License, which permits unrestricted use, distribution, and reproduction in any medium, provided the original work is properly cited.

\begin{abstract}
Organic light-emitting diode fabrication is suffering from extremely high material wasting during deposition especially using a typical point or even line source. Moreover, the need of depositing a high number of emitters and host(s) with a precise composition control in a single layer makes traditional vapor codeposition systems nearly impossible, unless otherwise with a very low yield. To improve, we have developed a novel thin-film deposition system with a planar source loadable with any premetered solventmixed organic compounds, plausibly with no component number limitation. We hence demonstrate experimentally, along with a Monte Carlo simulation, in the report the feasibility of using the technique to deposit on a large area-size substrate various organic materials with a relatively high material utilization rate coupling with high film uniformity. Specifically, nonuniformity of less than $\pm 5 \%$ and material utilization rate of greater than $70 \%$ have been obtained for the studied films.
\end{abstract}

\section{Introduction}

Organic light-emitting diodes (OLEDs) emit light when electricity is applied. They are used to create beautiful digital displays and also possible for use in solid-state lighting applications in the near future [1-3]. However, they are not the mainstream technologies yet because of the high manufacturing cost. To resolve, some key technologies need to be significantly improved, if not totally replaced.

Amongst organic thin-film deposition process is one key technology in OLEDs manufacturing. Most in use are evaporation systems equipped with a point source. Although the point source provides good film nonuniformity, in case the source-to-substrate distance is sufficiently long, the material utilization rate is known to be much less than $10 \%[4,5]$. The high waste of those expensive organic materials is one major cause of the current high cost in OLEDs manufacturing. This in turn obstructs the market acceptance. The problem becomes more severe as large areasize panels are demanded. To overcome, we propose a novel evaporation system equipped with a planar source, loadable with any premetered solvent-mixed organic compounds. It is a promising technology that can markedly improve the material utilization rate and easily scale up for deposition of large area-size substrate to enable low-cost manufacturing for OLED devices. In current study, we demonstrate a successful OLED device manufacturing by using in part the newly developed system. The new system has shown a better organic film uniformity and a higher material utilization than the conventional ones.

To assist the design of the new system, a parallel direct simulation Monte Carlo (DSMC) method [6] has been adopted in order to attain high film uniformity on a large area-size substrate for various different organic materials. The DSMC [6] method can be applied in several applications, including semiconductor related fabrication processes, fluid/thermal analysis of 


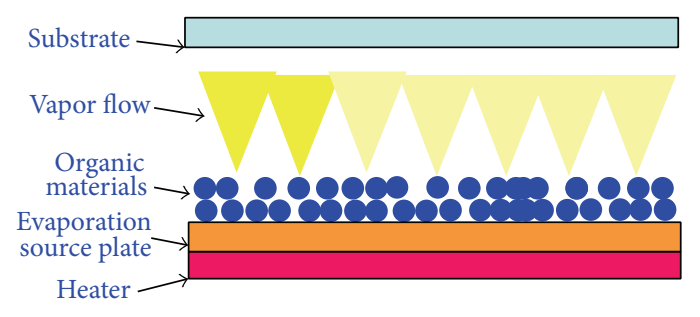

(a)

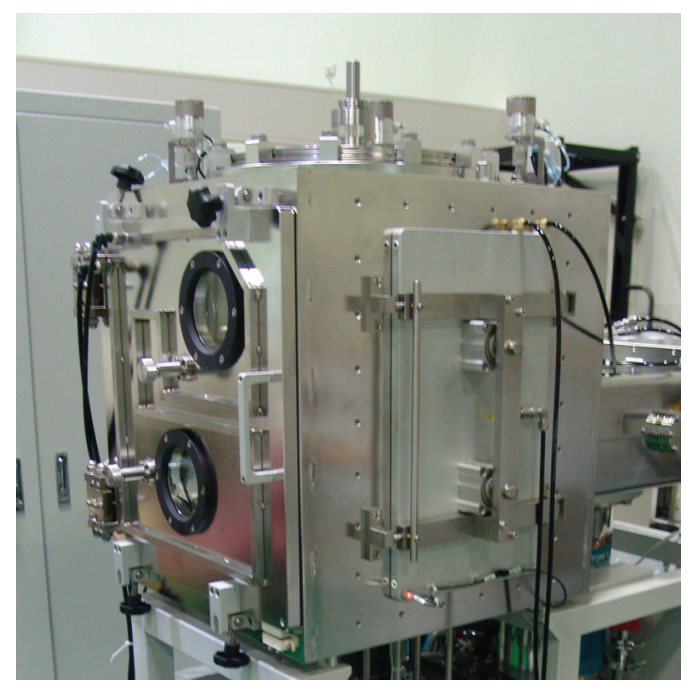

(b)

FIGURE 1: Illustration of (a) the concept and (b) photograph of the novel evaporation system.

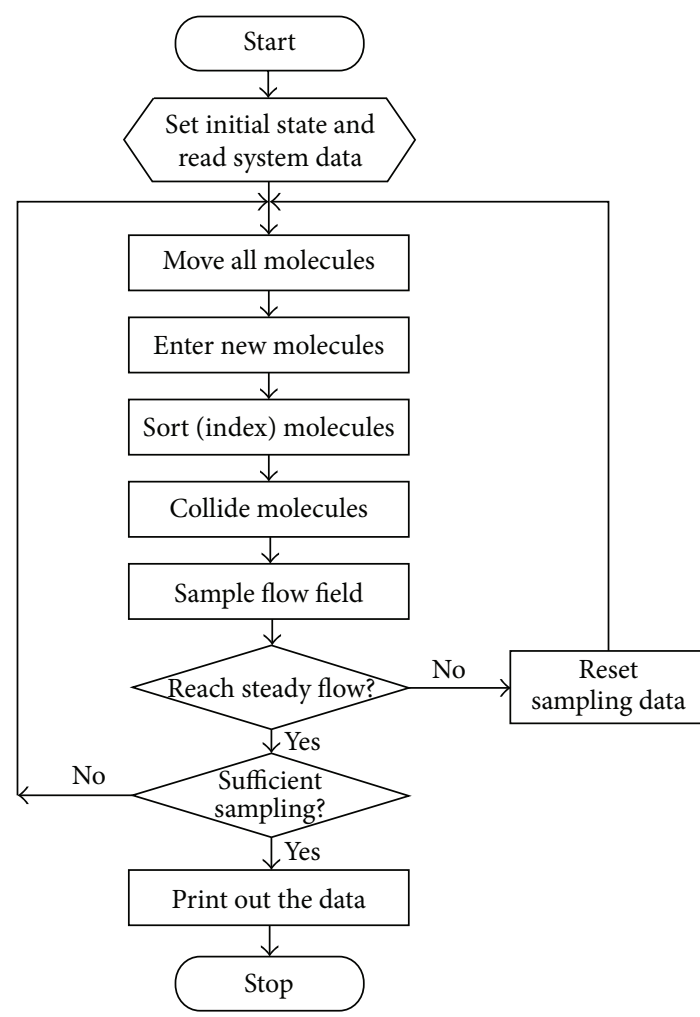

(a)

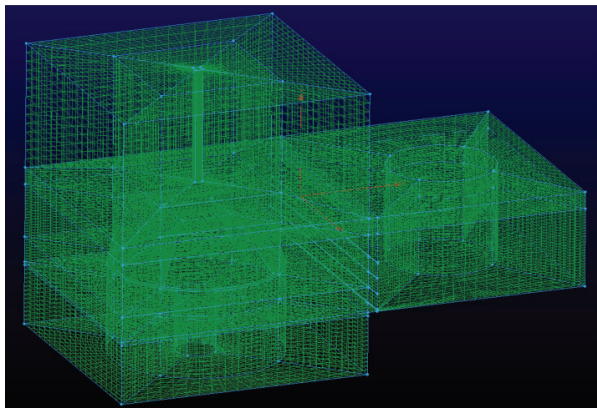

(b)

FIGURE 2: (a) Flow chart of the 3D DSMC method and (b) the 3D simulation cells.

MEMS/NEMS devices, hypersonic flow analysis, estimation of spacecraft, and scramjet engines flow. Importantly, it can also be adopted for modeling thin-film deposition process, such as, in the present case, the physical vapor evaporation of OLED, to obtain high uniformity on large area-size substrates for mixed compounds with any designated concentration [7-10].
It is learned that, via an accurate DSMC modeling, the development cost of the system can be tremendously reduced and the operational conditions of which can be rapidly optimized. It is also observed that the film uniformity is greatly improved and material utilization rate is markedly enhanced as the target-to-source (T/S) distance is decreased. 


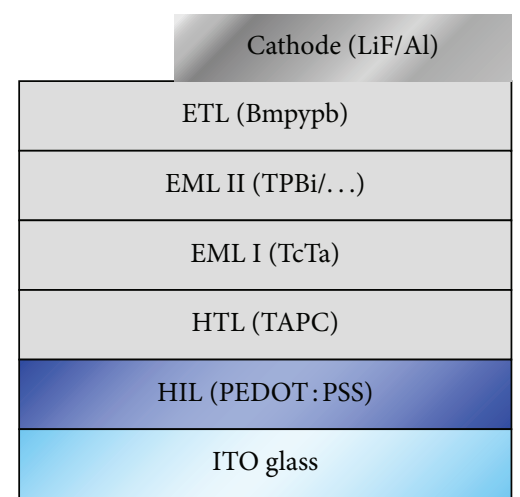

FIGURE 3: The structure of OLED device manufactured.

\section{Experimental}

2.1. Novel Evaporation System Equipped with a Planar Source Plate. The planar source module is illustrated in Figure 1(a) [11]. First, organic materials are deposited on the planar source plate by using a coating process. The planar source plate is then placed in a chamber. The heater under the planar source plate heats to the desired temperature, and the organic materials on the planar source plate are deposited upward onto the glass substrate. A shutter plate is set between the planar source plate and substrate to prevent the impurity from evaporating to the substrate at the initial time of the evaporation. The chamber is pumped to base pressure by using a cryopump equipped with a controlled gate valve. Figure 1(b) presents a photograph of the novel evaporation system equipped with a planar source plate.

\subsection{Parallel Direct Simulation Monte Carlo Method. The} direct simulation Monte Carlo (DSMC) method involves directly simulating particle collision kinetics for solving the Boltzmann equation and can automatically capture nonequilibrium phenomena without any convergence problem. This method has been widely used for simulating gas flow in rarefied gas dynamics. In addition, the parallel DSMC method can mitigate the problem of high computing power and increased computational time by simulating a large number of particles [12-15].

We applied parallel DSMC for modeling the transport phenomena and film deposition in an OLED processing chamber containing the planar source plate. Although numerous particles are generated in the flow field to represent real physical gas molecules, the DSMC algorithm can be readily parallelized using physical domain decomposition. Each processor executes the DSMC algorithm in sequence for all of the particles and cells of the computational grid, which are distributed among the processors, in its domain. Data communication occurs when particles cross the domain (processor) boundaries and are then transferred between processors.

Figure 2(a) presents a simplified flow chart of the three-dimensional (3D) DSMC method used in this study. Figure 2(b) presents the 3D simulation cells of the planar source system. Table 1 lists the properties of Organic Material
TABle 1: The properties of Organic Material A.

\begin{tabular}{lc}
\hline Item & Value \\
\hline Molecular weight & 459.43 \\
Molecular mass $(\mathrm{kg})$ & $7.63 E-26$ \\
Ref. temperature $(\mathrm{K})$ & 273 \\
Surface temperature $\left({ }^{\circ} \mathrm{C}\right)$ & 155 \\
Surface temperature $(\mathrm{K})$ & 428 \\
Flux $J\left(\mathrm{~mole}^{2} / \mathrm{m}^{2} \cdot \mathrm{s}\right)$ & $8.07 E-10$ \\
Flux $n\left(\# / \mathrm{m}^{2} \cdot \mathrm{s}\right)$ & $4.86 E+14$ \\
Mean thermal speed $(\mathrm{m} / \mathrm{s})$ & $4.44 E+02$ \\
Number density $n\left(\# / \mathrm{m}^{3}\right)$ & $1.09 E+12$ \\
Degrees of freedom $\xi$ & 3 \\
Viscosity coefficient & $3.29 E-05$ \\
Viscosity index $\omega$ & 1.63 \\
Reference diameter $d(\mathrm{~m})$ & $1.76 E-09$ \\
Mean free path $\lambda$ & $1.50 E+02$ \\
\hline
\end{tabular}

A [11]. According to the simulation result, we modified the planar source plate used in this study to achieve optimal performance.

2.3. Methods and Device Fabrication. The thickness of the Alq3 thin films deposited using the planar source plate was measured to assess the nonuniformity of the film thickness. We deposited organic material $(5,6,11,12)$ tetraphenylnaphthacene (rubrene) onto the glass substrate at varying distances between the target substrate and planar source plate $(\mathrm{T} / \mathrm{S})$ to measure the material utilization of the planar source plate.

Figure 3 presents the structure of the fabricated OLED device. The substrate size is $5 \times 5 \mathrm{~cm}$.

First, we fabricated a hole-injection layer (HIL) on a precleaned indium tin oxide (ITO) anode by spin-coating an aqueous solution of poly(3,4-ethylene-dioxythiophene)-poly (styrenesulfonate) (PEDOT:PSS) in nitrogen.

The hole-transport layer (HTL) was then deposited using the proposed system equipped with the planar source plate. We used di-[4-(N,N-ditolyl-amino)-phenyl]cyclohexane (TAPC) as the hole-transport material. The TAPC was dissolved in tetrahydrofuran, spray-coated onto the planar source plate at room temperature in air, and dried. The heater heated to $450^{\circ} \mathrm{C}$, and the TAPC was deposited upward onto the glass substrate exhibiting a T/S gap of $5 \mathrm{~cm}$ at a pressure of $1.33 \mathrm{~Pa}$.

The other organic and metal layers were prepared using a conventional system at approximately $1.33 \times 10^{-3} \mathrm{~Pa}$. A $4,4^{\prime}, 4^{\prime \prime}$-tris(carbazol-9-yl)-triphenylamine (TcTa) was used as the host with a small amount of blue dopant for the emissive layer (EML) I. A 1,3,5-tris(1-phenyl-1 h-benzimidazol-2yl) benzene (TPBi) was used as host with a small amount of red dopant, yellow dopant, and green dopant for the EML II. Subsequently, 1,3-bis[3,5-di (pyridin-3-yl) phenyl]benzene (Bmpypb) was prepared to form an electron-transport layer (ETL). 


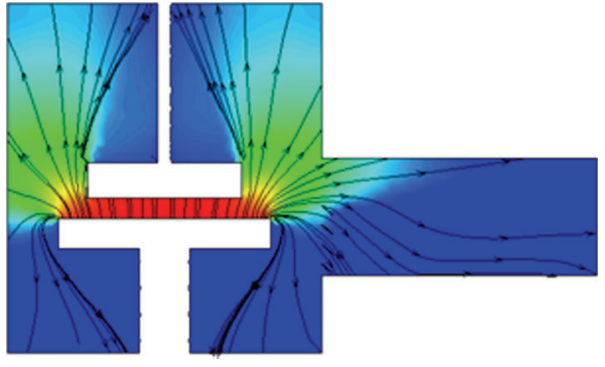

(a)

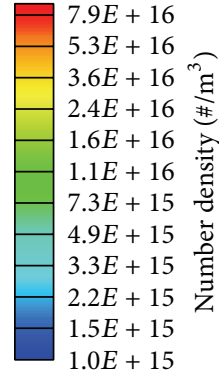

$1.0 E+15$

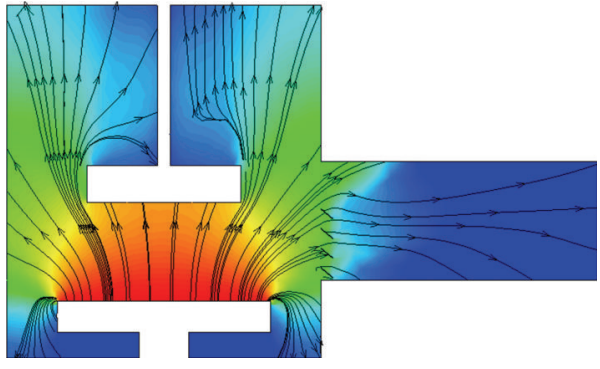

(b)

FIGURE 4: Number density distribution diagrams with (a) $30 \mathrm{~mm}$ and (b) $150 \mathrm{~mm}$ T/S distance (slice from $x-y$ plane).

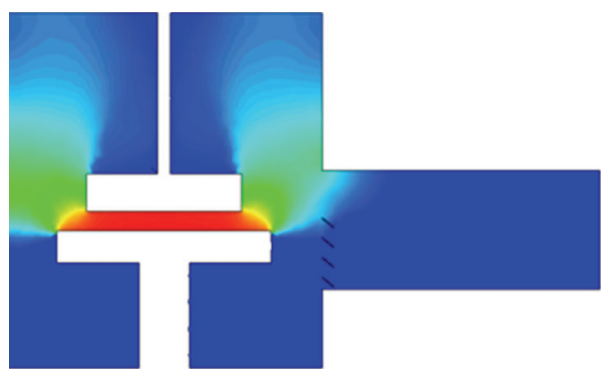

(a)

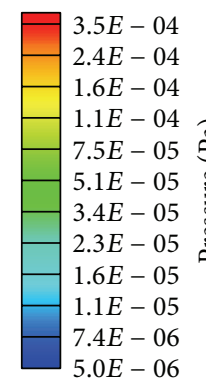

.0E -06

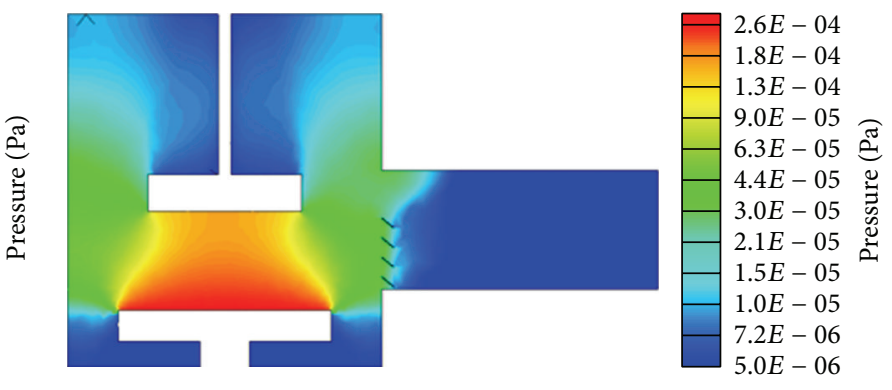

(b)

FIGURE 5: Pressure distribution diagrams with (a) $30 \mathrm{~mm}$ and (b) $150 \mathrm{~mm}$ T/S distance (slice from $x-y$ plane).

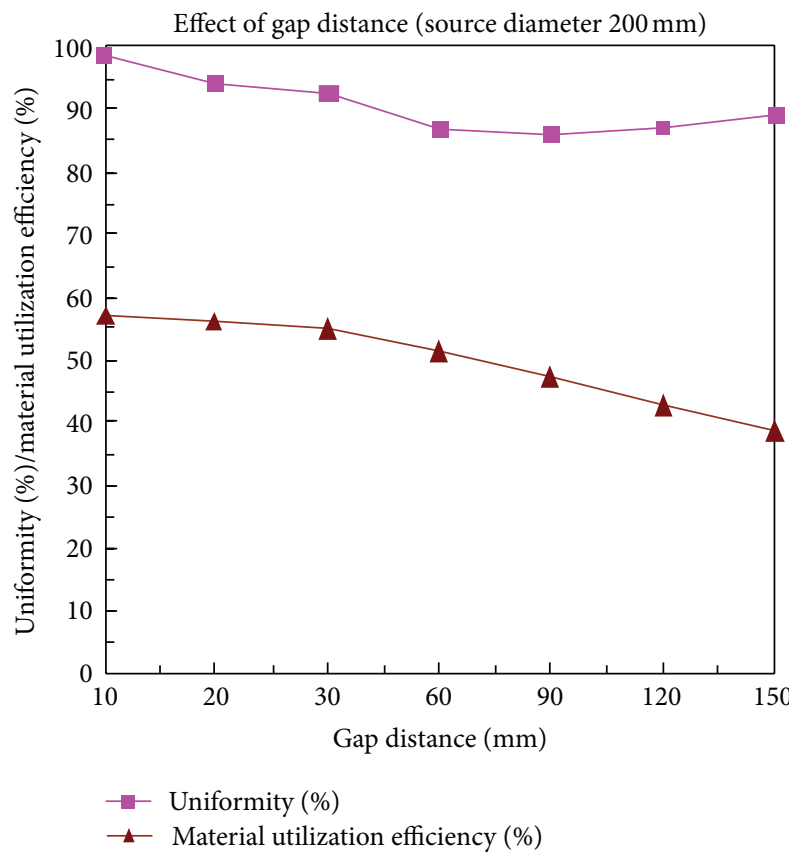

Figure 6: The relationship of thin-film uniformity and material utilization rate of Organic Material A at varying T/S gap distances.

Subsequently, an electron-injection layer (EIL) of lithium fluoride $(\mathrm{LiF})$ and aluminum cathode was deposited using thermal evaporation at $1.33 \times 10^{-3} \mathrm{~Pa}$.

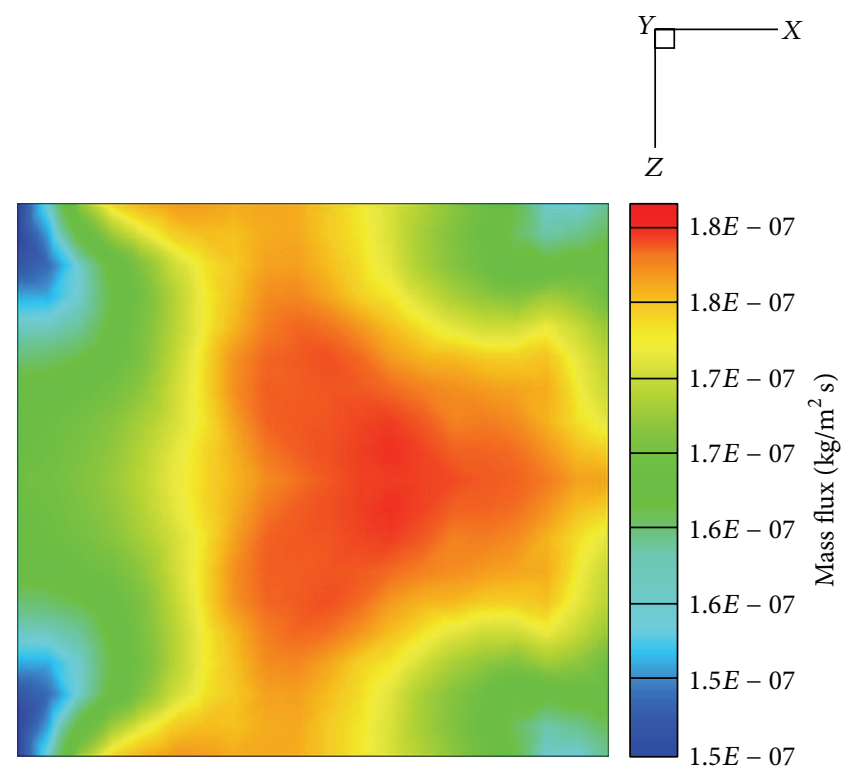

Figure 7: Mass flux of Organic Material A.

Luminance, chromaticity, and electroluminescence spectra of the OLEDs were recorded using a PR-650 SpectraScan Colorimeter. The current-voltage characteristics were measured using a Keithley 2400 source meter. All of the measurements were performed at room temperature in air. 
TABle 2: Thickness of the "Alq3" thin films deposited by the planar source.

\begin{tabular}{lcccccccc}
\hline Position & 1 & 2 & 3 & 4 & 5 & 6 & 7 & 8 \\
\hline Thickness $(\mathrm{nm})$ & 151.71 & 149.59 & 150.82 & 153.53 & 161.03 & 154.64 & 152.15 & 158.46 \\
\hline
\end{tabular}

TABLE 3: Material utilization rate of the planar source.

\begin{tabular}{lccc}
\hline T/S $(\mathrm{cm})$ & $\begin{array}{c}\text { Planar source plate weight } \\
\text { difference }(\mathrm{mg})\end{array}$ & $\begin{array}{c}\text { Substrate weight difference } \\
(\mathrm{mg})\end{array}$ & $\begin{array}{c}\text { Material utilization rate }(\%) \\
14\end{array}$ \\
\hline 14.2 & 4.7 & 33.1 \\
12 & 13.6 & 5.7 & 41.9 \\
10 & 5.4 & 4.2 & 77.8 \\
\hline
\end{tabular}

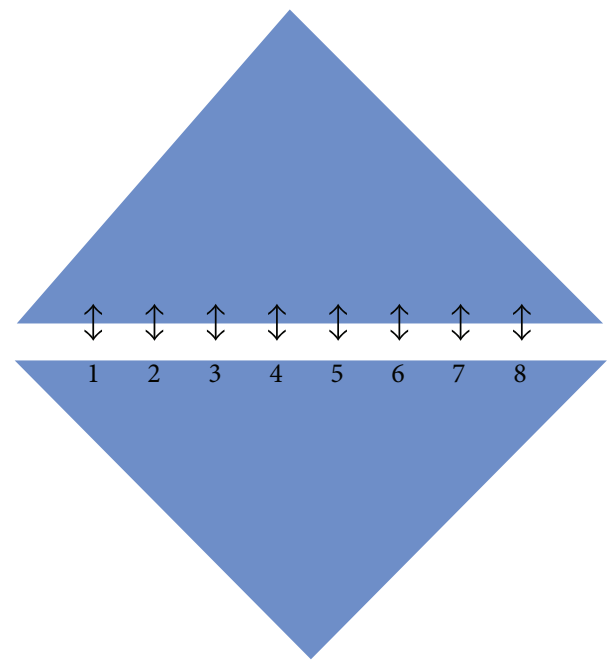

FIGURE 8: The thickness measurement positions of the substrate.

\section{Results and Discussion}

3.1. Parallel Direct Simulation Monte Carlo Method. Figure 4 presents the number density contour for observing the distribution from the evaporation source to the substrate. The flux of molecules from the evaporation source to the substrate is uniform when the T/S distance is equal to $30 \mathrm{~mm}$. Figure 5 illustrates the pressure distribution contour for a T/S distance of (a) $30 \mathrm{~mm}$ and (b) $150 \mathrm{~mm}$ (slice from the $x$-y plane). The pressure distribution at a T/S distance of $30 \mathrm{~mm}$ is more uniform than that at a T/S distance of $150 \mathrm{~mm}$ [11], because tilted blade gate openings, as indicated by the " $\mid$ " in Figures 4 and 5, were constructed to obstruct the flow to reduce the influence of the asymmetrical pumping system. Because the T/S gap was short and the pressure was considerably low, the influence of the asymmetrical system was reduced. Although the molecules number density and the pressure distribution were not symmetrical near the outlet region, both were symmetrical in the region of the substrate and the target. High film uniformity was thus obtained.

Figure 6 presents the relationship of thin-film uniformity and material utilization rate of Organic Material A at varying $\mathrm{T} / \mathrm{S}$ gap distances. The uniformity of the thin film is more than $85 \%$ when the T/S distance ranges from $30 \mathrm{~mm}$ to
$150 \mathrm{~mm}$. The thin-film thickness uniformity and material utilization rate are high when the T/S distance is decreased, which differs from the conventional thermal evaporation system equipped with a point source.

The film uniformity can be estimated according to the mass flux of the particles passing through the substrate surface, as illustrated in Figure 7. As indicated, the mass flux is distributed from $1.5 \times 10^{-7}$ to $1.8 \times 10^{-7} \mathrm{~kg} / \mathrm{m}^{2} \mathrm{~s}$, with most of the mass flux centered from $1.6 \times 10^{-7}$ to $1.8 \times 10^{-7} \mathrm{~kg} / \mathrm{m}^{2}$ s. Equations (1)-(3) are used to calculate the nonuniformity. Because of the high uniformity of particle distribution, the calculated film nonuniformity is only $3.5 \%$. Consider

$$
\begin{gathered}
\bar{T}=\frac{1}{N} \sum_{i=1}^{N} t_{i} \\
\Delta t=\sqrt{\frac{1}{n-1} \sum_{i=1}^{N}\left(t_{i}-\bar{T}\right)^{2}} \\
\mathrm{UN}=\frac{\Delta t}{\bar{T}} \times 100 \%,
\end{gathered}
$$

where $\bar{T}$ is the mass flux average, $N$ is the total mass flux, $t_{i}$ is the mass flux, $\Delta t$ is the standard deviation of mass flux, and $\mathrm{UN}$ is the nonuniformity number.

3.2. Nonuniformity of the Film Thickness. The thin films composed of tris(8-hydroxy-quinolinato) aluminum (Alq3) were deposited using the planar source plate at a distance of $5 \mathrm{~cm}$ between the target substrate and planar source plate (T/S).

The thickness of the Alq3 thin films was measured using the Dektak XT Surface Profiler, as indicated in Table 2 [11]. The positions measured from the $10 \times 10 \mathrm{~cm}$ glass substrate are presented in Figure 8. The nonuniformity of the film thickness can be determined using (4). Consider

$$
\text { Nonuniformity }( \pm \%)=\frac{\left(T_{\max }-T_{\min }\right)}{2 T_{\mathrm{avg}}} \times 100 \% \text {, }
$$

where $T_{\max }, T_{\min }$, and $T_{\text {avg }}$ are the maximal, minimal, and average values of the film thickness, respectively. According to (4), the nonuniformity of the Alq3 thin-film thickness was 


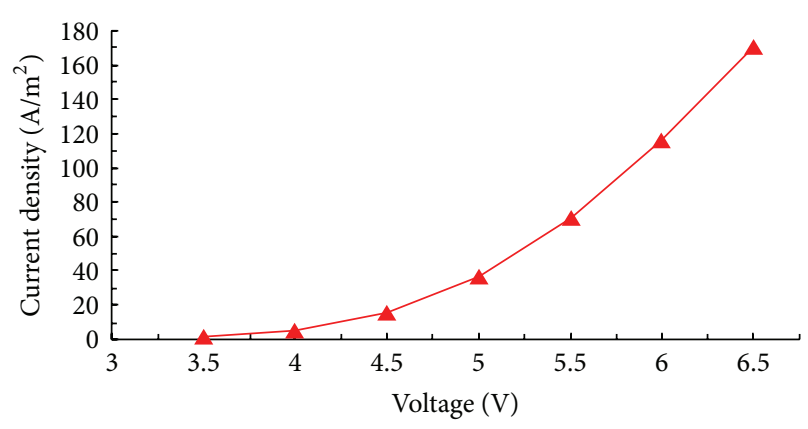

(a)

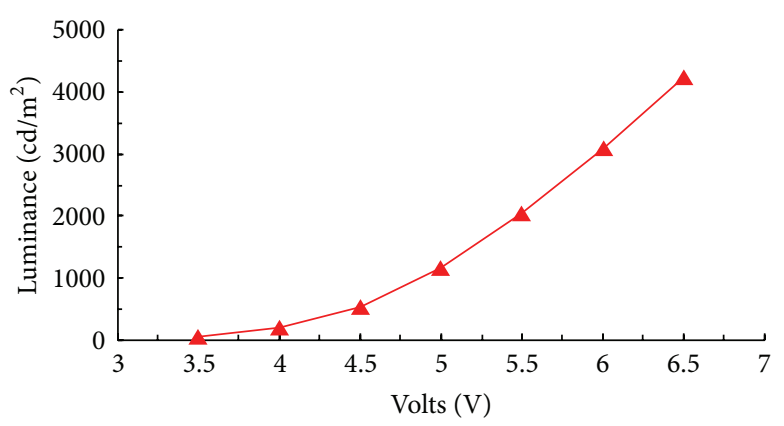

(b)

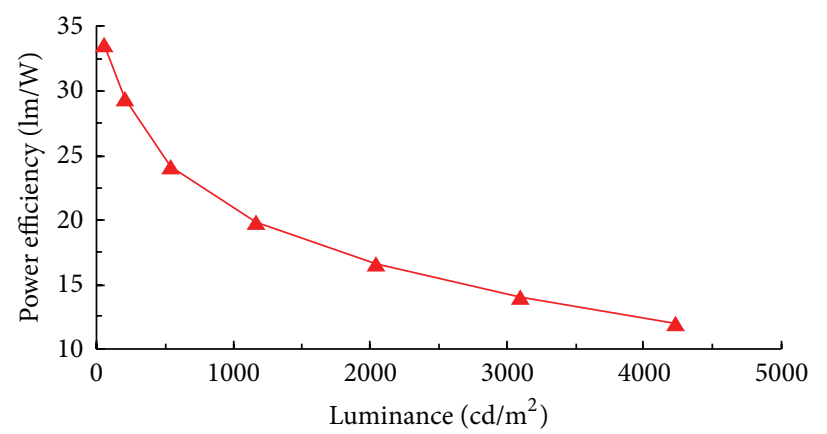

(c)

FIgURE 9: (a) The current density-voltage characteristic. (b) The brightness-voltage characteristic. (c) The power efficiency versus luminance of the OLED device.

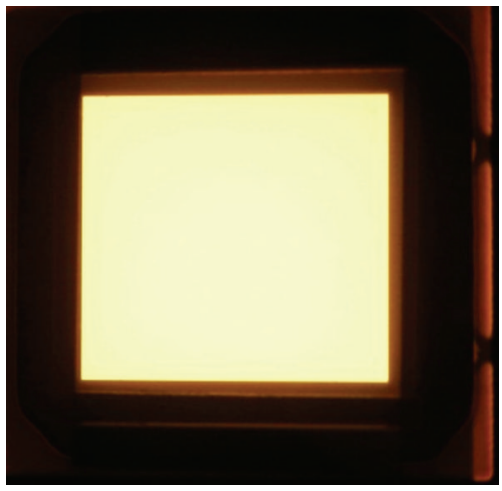

FIgUre 10: The OLED device illuminated at $3.5 \mathrm{~V}$.

$\pm 3.7 \%$. The uniformity can be easily attained using the planar source plate at a short T/S distance [11].

A layer using a host and a blue dye to obtain a film nonuniformity of $\pm 2.7 \%$ was successfully achieved. Specifically, the film was prepared using the solvent premixing deposition method [16-19]. The film was deposited shot by shot by using the planar source. For each shot, the precise doping concentration with a favorable reproducibility was obtained by controlling the amount of solvent-mixed premetered organic compounds. To the moment, no substantial residual materials, either single coating materials (HTL) or two materials (host and blue dye), were observed on the planar source after evaporation. This meant that most of the prepared solvent premixing materials, including the dopant and the host, were successfully evaporated during the heating process. The conventional codeposition doping process requires an extremely low deposition rate and a highly precise deposition-time control, which is impractical and occasionally impossible. Insufficient doping or overdoping of one or more of the dyes, such as red or green dyes, frequently occurs, explaining why numerous previously reported white OLEDs have failed to yield pure white emission [16].

3.3. Material Utilization Rate. To measure the material utilization rate of the planar source plate directly, $(5,6,11,12)$ tetraphenylnaphthacene (rubrene) was deposited onto the glass substrate at varying distances between the target substrate and planar source plate (T/S). We used approximately $16 \mathrm{mg}$ of rubrene for each studied material in the planar source. The weight difference of the planar source plate and glass substrate was measured, as indicated in Table 3 [11]. When the T/S distance was $10 \mathrm{~cm}$, the material utilization was as high as $77.8 \%$. Based on the experimental results, the T/S distance should be as short as possible to increase the material utilization rate in the deposition processes.

A $77.8 \%$ material utilization rate was obtained using the newly developed planar source, which was much higher than the $3 \%$ to $5 \%$ rate observed when using typical point sources. Improving material utilization by a wide margin should decrease the manufacturing cost of mass production.

3.4. Characterization of the Device. We successfully fabricated OLED devices by using the HTL deposited 

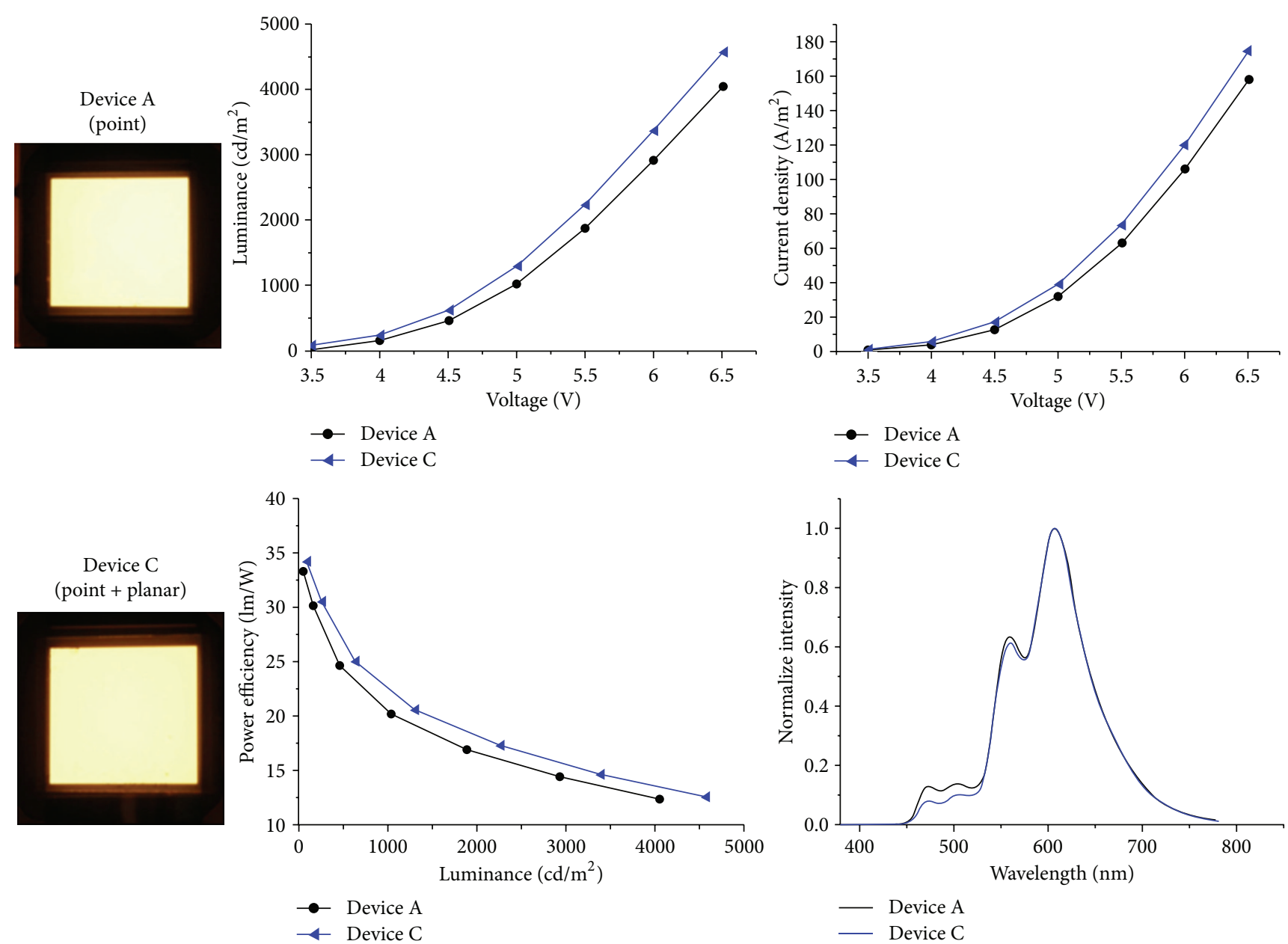

FIGURE 11: The device performance compared with that of a device prepared using a complete conventional point source.

using the proposed system equipped with a planar source plate and the other layers deposited using a conventional system.

Figure 9(a) presents the current density-voltage characteristic and Figure 9(b) presents the brightness-voltage characteristic. The current density was $31 \mathrm{~A} / \mathrm{m}^{2}$ and the brightness was $1000 \mathrm{~cd} / \mathrm{m}^{2}$ at an operation voltage of $4.85 \mathrm{~V}$. Figure $9(\mathrm{c})$ presents the power efficiency versus luminance of the OLED device. The power efficiency at $1000 \mathrm{~cd} / \mathrm{m}^{2}$ is $21.1 \mathrm{~lm} / \mathrm{W}$. The device also exhibits a color rendering index (CRI) of 70 at $1000 \mathrm{~cd} / \mathrm{m}^{2}$. Figure 10 presents a photograph of the OLED device illuminated at $3.5 \mathrm{~V}$.

Figure 11 presents the device performance compared with that of a device prepared by using a complete conventional point source. The characteristics of luminance, current density, and power efficiency, achieved by partially using the proposed system equipped with a planar source, were higher than those achieved using a complete conventional point source. The wavelength spectra were similar in both cases.

\section{Conclusion}

A new system designed with a planar source is manufactured for OLED application. A parallel DSMC method is used for modeling the process of organic material deposition of the planar source system. As the T/S distance is decreased, the film uniformity is higher, and the material utilization rate is also higher, much different from the point source based evaporation system. We can reduce the cost of the planar source development and optimize the operating conditions through accurate DSMC modeling. Using such a planar source has at least three advantages, namely, a high material utilization rate, high film uniformity, and high degree of device design freedom. To the moment, we successfully achieved a layer with a host and a blue dye with a film nonuniformity of $\pm 2.7 \%$. Specifically, the film was prepared using the solvent premixing deposition method. Moreover, by using the newly developed planar source, a $77.8 \%$ material utilization rate was obtained, which is much higher than the $3 \%$ to $5 \%$ rate observed when using the typical point source. The system has enabled the organic thin films to be deposited 
with a less than $5 \%$ nonuniformity and a material utilization rate of over $70 \%$. We successfully demonstrate the fabrication of an OLED device with the hole transporting layer deposited by the new system with the planar source and the other layers deposited by the conventional system. The power efficiency of the OLED device is $21.1 \mathrm{~lm} / \mathrm{W}$ with a CRI of 70 at 1,000 $\mathrm{cd} / \mathrm{m}^{2}$. The evaporation system equipped by the novel planar source can obtain large-area uniformity for thin-film evaporation and high material utilization rate of various organic materials.

\section{Conflict of Interests}

The authors declare that they have no conflict of interests regarding the publication of this paper.

\section{Acknowledgments}

The authors acknowledge Professor Jong-Shinn Wu and his laboratory team of the Department of Mechanical Engineering, National Chiao Tung University, for assisting with the DSMC method. This study was financially supported by the Taiwan Ministry of Economic Affairs through Grants nos. 102-EC-17-A-05-01-1111, D301AR3B00, and 102-EC-17-A-07S1-181.

\section{References}

[1] S. Reineke, F. Lindner, G. Schwartz et al., "White organic lightemitting diodes with fluorescent tube efficiency," Nature, vol. 459, no. 7244, pp. 234-238, 2009.

[2] S. J. Su, E. Gonmori, H. Sasabe, and J. Kido, "Highly efficient organic blue-and white-light-emitting devices having a carrierAnd exciton-confining structure for reduced efficiency roll-off," Advanced Materials, vol. 20, no. 21, pp. 4189-4194, 2008.

[3] T. H. Han, Y. Lee, M. R. Choi et al., "Extremely efficient flexible organic light-emitting diodes with modified graphene anode," Nature Photonics, vol. 6, no. 2, pp. 105-110, 2012.

[4] C. C. Hwang, "Plane source and in-line deposition system for OLED manufacturing," in Proceedings of the 44th International Symposium, Seminar, and Exhibition (SID '06), pp. 1542-1545, June 2006, Technical Papers 37.

[5] H. W. Kim, S. Y. Han, H. B. Shim et al., "Improvement of material utilization of organic evaporation source for manufacturing large-sized AMOLED devices," in Proceedings of the International Symposium, Seminar, and Exhibition (SID '08), pp. 1450-1453, May 2008, Technical Papers 39.

[6] G. A. Bird, Molecular Gas Dynamics and the Direct Simulation of Gas Flows, Clarendon Press, Oxford, UK, 1994.

[7] D. M. Mattox, Handbook of Physical Vapor Deposition (PVD) Processing, Noyes, Westwood, NJ, USA, 1998.

[8] M. Baldo, M. Deutsch, P. Burrows et al., "Organic vapor phase deposition," Advanced Materials, vol. 10, no. 18, pp. 1505-1514, 1998.

[9] H. Fukumoto, Y. Muramatsu, T. Yamamoto, J. Yamaguchi, K. Itaka, and H. Koinuma, "Combinatorial physical vapor deposition of $\pi$-conjugated organic thin film libraries," Macromolecular Rapid Communications, vol. 25, no. 1, pp. 196-203, 2004.
[10] H. Usui, "Formation of polymer thin films and interface control by physical vapor deposition," in Proceedings of the SPIE, vol. 7404, August 2009.

[11] S.-H. Lai, C-C. Chen, C.-C. Wang, F.-C. Tung, S.-H. Chen, and Y.-S. Wang, "OLED deposition system using plane-source evaporation techniques," in Proceedings of the 20th International Display Workshops (IDW'13), 2013.

[12] J.-S. Wu and Y.-Y. Lian, "Parallel three-dimensional direct simulation Monte Carlo method and its applications," Computers and Fluids, vol. 32, no. 8, pp. 1133-1160, 2003.

[13] J. S. Wu and K. C. Tseng, "Parallel DSMC method using dynamic domain decomposition," International Journal For Numerical Methods in Engineering, vol. 63, no. 1, pp. 37-76, 2005.

[14] J.-S. Wu, K.-C. Tseng, and F.-Y. Wu, "Parallel three-dimensional DSMC method using mesh refinement and variable time-step scheme," Computer Physics Communications, vol. 162, no. 3, pp. $166-187,2004$.

[15] C.-C. Su, K.-C. Tseng, H. M. Cave et al., "Implementation of a transient adaptive sub-cell module for the parallel DSMC code using unstructured grids," Computers \& Fluids, vol. 39, no. 7, pp. 1136-1145, 2010.

[16] J. H. Jou, Y. S. Chiu, C. P. Wang, R. Y. Wang, and H.-C. Hu, "Efficient, color-stable fluorescent white organic light-emitting diodes with single emission layer by vapor deposition from solvent premixed deposition source," Applied Physics Letters, vol. 88, no. 19, Article ID 193501, 2006.

[17] Y. C. Tsai and J. H. Jou, "Long-lifetime, high-efficiency white organic light-emitting diodes with mixed host composing double emission layers," Applied Physics Letters, vol. 89, no. 24, Article ID 243521, 2006.

[18] J.-H. Jou, C.-Y. Hsieh, P.-W. Chen, S. Kumar, and J. H. Hong, "Candlelight style organic light-emitting diode: a plausibly human-friendly safe night light," Journal of Photonics for Energy, vol. 4, no. 1, Article ID 043598, 2014.

[19] J. H. Jou, C. P. Wang, M. H. Wu et al., "Efficient fluorescent white organic light-emitting diodes with blue-green host of di(4-fluorophenyl)amino-di(styryl)biphenyl," Organic Electronics: Physics, Materials, Applications, vol. 8, no. 1, pp. 2936, 2007. 

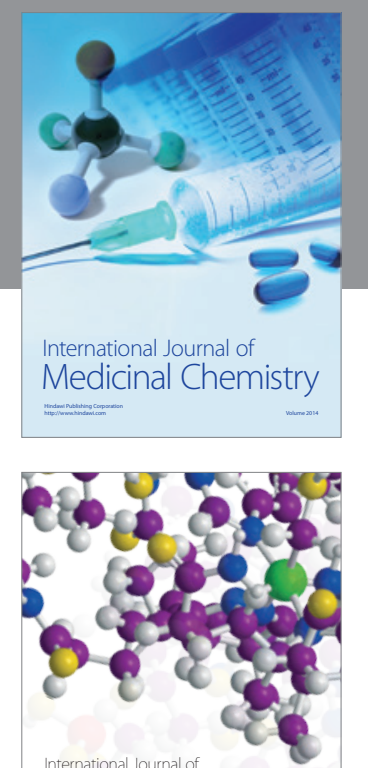

\section{Carbohydrate} Chemistry

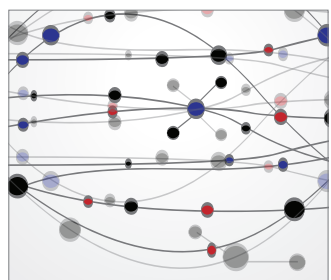

The Scientific World Journal
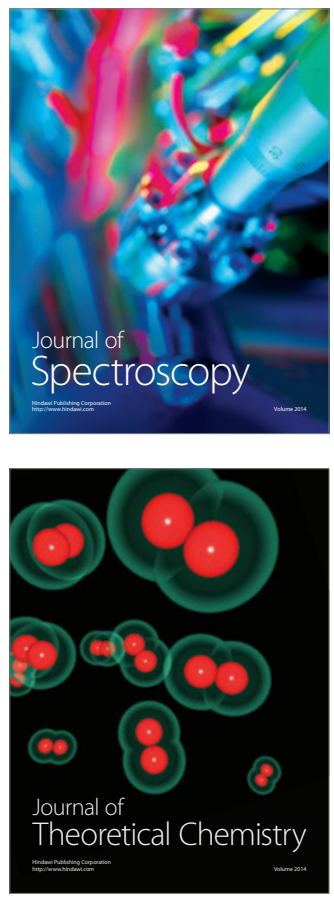
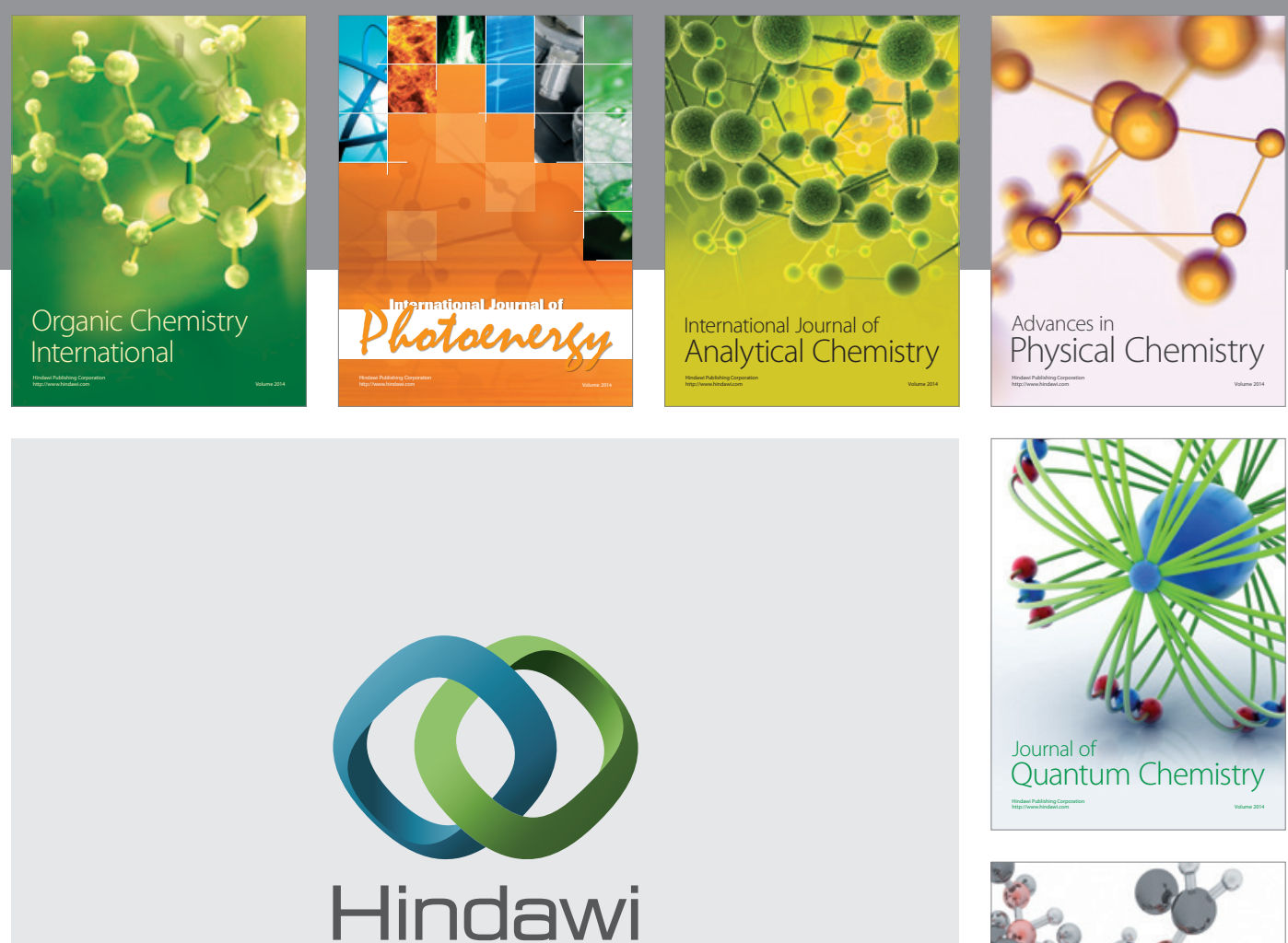

Submit your manuscripts at

http://www.hindawi.com

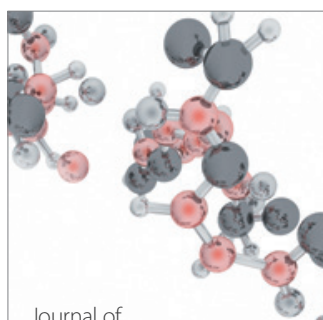

Analytical Methods

in Chemistry

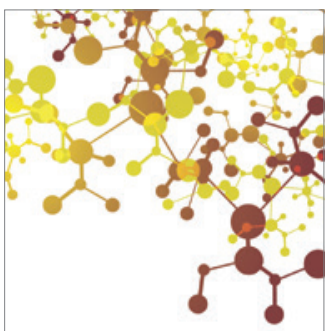

Journal of

Applied Chemistry

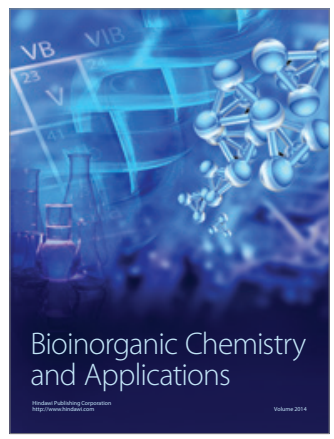

Inorganic Chemistry
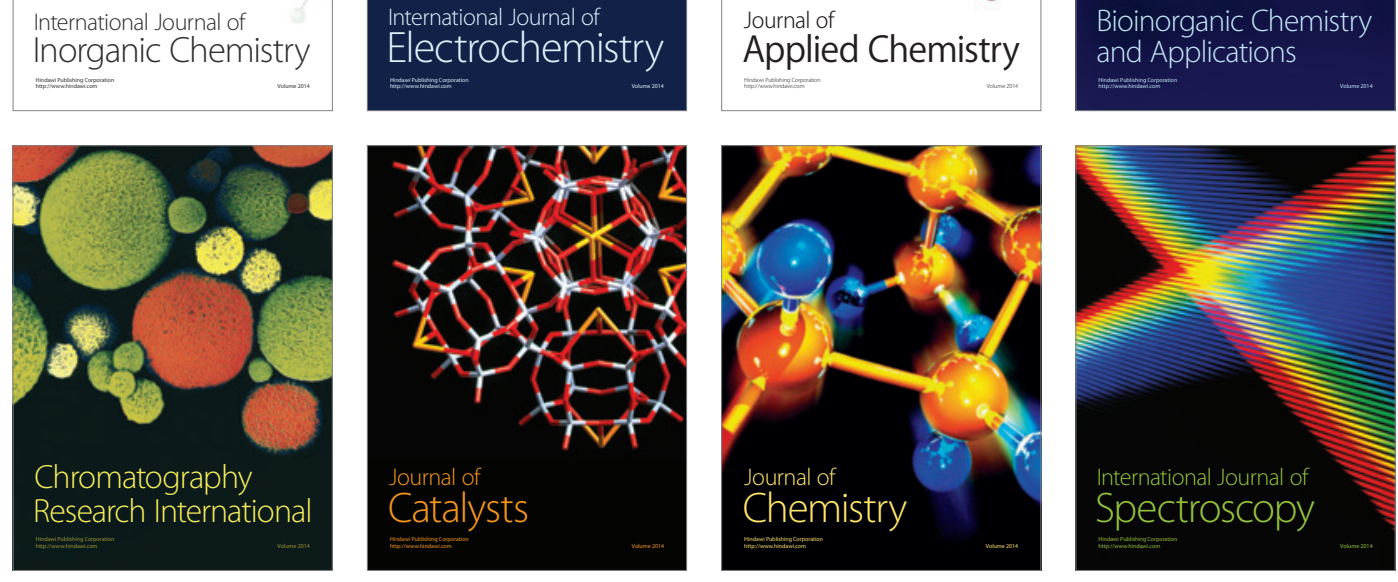